UNA MIRADA LONGITUDINAL Y TRANSGENERACIONAL SOBRE LOS CONFLICTOS ENTRE MADRES Y ADOLESCENTES.

ÁGUEDA PARRA JIMÉNEZ Y ALFREDO OLIVA DELGADO

DEPARTAMENTO DE PSICOLOGÍA EVOLUTIVA Y DE LA EDUCACIÓN

UNIVERSIDAD DE SEVILLA 
Dirección de correspondencia:

Águeda Parra Jiménez

Dpto. Psicología Evolutiva y de la Educación

Universidad de Sevilla

Camilo José Cela s/n, 41018, Sevilla.

Tfno.: 954554333

Fax: 954557642

Correo electrónico: aparra@us.es 


\section{UNA MIRADA LONGITUDINAL Y TRANSGENERACIONAL SOBRE LOS CONFLICTOS ENTRE MADRES Y ADOLESCENTES.}

\section{RESUMEN}

Los conflictos familiares durante la adolescencia han generado muchas investigaciones en los últimos años, sin embargo, son pocas las que han usado diseños longitudinales y han tenido en cuenta la opinión de las madres. Los objetivos de este trabajo son dos, analizar la evolución de la frecuencia de los conflictos a lo largo de la adolescencia, y comparar las perspectivas de madres y jóvenes. Para cubrir el primero de ellos analizamos la estabilidad absoluta y relativa de la frecuencia de los conflictos, así como las trayectorias seguidas por grupos de sujetos. Entre los resultados más destacados podemos señalar la estabilidad en la frecuencia de los conflictos, ya que la mayoría de los adolescentes no percibe grandes cambios en las discusiones con sus madres -aunque respecto a temas concretos sí aparecen diferencias-. Por otro lado, las madres perciben menos conflictos que sus hijos e hijas, y han aparecido interesantes diferencias de género.

Palabras clave: adolescencia, relaciones familiares, conflictos entre madres y adolescentes. 
A LONGITUDINAL AND TRANSGENERATIONAL VIEW OF MOTHERADOLESCENT CONFLICTS.

\begin{abstract}
Family conflicts during adolescence have been studied in depth over the past years, though only a few studies have used longitudinal designs and taken mothers' opinions into account. The purpose of the present study was 1) to analyse how frequency of conflicts evolve throughout adolescence, and 2) compare mothers and adolescents' views. We therefore analysed absolute and relative stability of conflict frequency and identified the trajectory followed by groups of subjects. Among the most salient result is the stability in conflict frequency; most adolescents do not perceive great changes in discussions with their mothers, though there were differences regarding specific topics. On the other hand, mothers perceive fewer conflicts than both their sons and daughters, and interesting gender differences have also emerged.
\end{abstract}

Key words: adolescence, family relationships, mother-adolescent conflicts. 


\section{UNA MIRADA LONGITUDINAL Y TRANSGENERACIONAL SOBRE LOS CONFLICTOS ENTRE MADRES Y ADOLESCENTES.}

\section{INTRODUCCIÓN}

La imagen social de las relaciones familiares durante la adolescencia está caracterizada por el conflicto entre padres, madres y jóvenes. Conflictos que tienden a disminuir con los años, cuando la dinámica familiar se normaliza y alcanza un nuevo equilibrio. Sin embargo, las investigaciones científicas aún no aportan datos concluyentes sobre esta trayectoria.

Diferentes investigaciones apuntan que coincidiendo con la pubertad aumentan los conflictos familiares y se produce un distanciamiento entre los adolescentes y sus madres y padres (Conger y Ge, 1999; Holmbeck y Hill, 1991; Steinberg, 1987; 1988). Autores como Peter Bloss (1979) consideran que cierta ruptura y distanciamiento en la relación con madres y padres es un requisito indispensable para el correcto funcionamiento del adolescente, ya que para establecerse como un adulto independiente, el chico y la chica debe separarse de sus padres tanto física como emocionalmente. El concepto de Individuación es central desde esta perspectiva, individuación que implica una desvinculación de los cuidadores a través de la cual chicos y chicas pueden "salir" del hogar familiar y lograr así unas relaciones afectivas positivas con otras personas. La rebelión hacia los padres y el aumento de los conflictos se convierten así en una consecuencia inevitable de esta etapa, que provoca una ruptura en los vínculos familiares y un obligado reajuste en las relaciones. 
Sin embargo, en lo que no existe tanto acuerdo es en la trayectoria que siguen los conflictos a lo largo de los años adolescentes. Tradicionalmente este cambio ha sido descrito en términos de una figura de $U$ invertida, con un aumento de la conflictividad entre la adolescencia inicial y media y una posterior disminución una vez llegada la adolescencia tardía (Montemayor, 1983; Paikoff y Brooks-Gunn, 1991). Sin embargo, Laursen, Coy y Collins en su meta-análisis publicado en 1998 y basado en 53 investigaciones no encuentran apoyo al modelo de la U invertida. Sus datos más bien apuntan a que con la edad se observa un decremento lineal en la frecuencia de los conflictos familiares.

Hay autores cuyos hallazgos no apoyan esta idea de la disminución de los conflictos con la edad (Dekovic, 1999; Bosma, Jackson, Zusling, Zani, Cicognani, Xerri, Honess, y Charman, 1996; Galambos y Almeida, 1992). Para ellos la trayectoria de los conflictos depende de los temas concretos objeto de discusión, ya que algunos emergerían como fuente de conflicto a medida que chicas y chicos van atravesando la adolescencia. Este sería el caso por ejemplo de los conflictos sobre la elección profesional (Bosma, et al., 1996), o sobre el empleo del dinero (Galambos y Almeida, 1992).

En cualquier caso, las discrepancias en las conclusiones probablemente puedan ser explicadas, al menos en parte, atendiendo a las diferencias metodológicas entre los estudios. Por un lado, no todos los trabajos utilizan la misma forma de evaluar el conflicto familiar (Holmbeck, Paikoff y Brooks-Gunn, 1995). Mientras que algunos se basan en el análisis de interacciones, otros parten de entrevistas, o analizan las formas de actuación de madres, padres y adolescentes ante situaciones conflictivas hipotéticas. Otra posible explicación a los datos contradictorios la encontramos en la fuente de información utilizada, ya que algunas investigaciones se basan en la información obtenida exclusivamente de los padres o de los adolescentes, mientras que otras se basan de las 
opiniones de ambos. Finalmente, los resultados inconsistentes también podrían ser debidos a la escasez de estudios longitudinales, ya que la mayoría de los trabajos parten de diseños transversales que no nos permiten concluir de forma clara acerca de los cambios internos en la dinámica familiar durante estos años (Conger y Ge, 1999).

Con respecto a los temas que provocan discusiones y riñas familiares, estudios realizados en diferentes países, incluyendo España, coinciden en afirmar que los conflictos más frecuentes son motivados por aspectos de la vida diaria como la forma de vestir, la hora de llegar a casa, las tareas del hogar o asuntos académicos (Arnett, 1999; Dekovic, 1999; Elzo et al., 1999; Noller, 1994; Parra y Oliva, 2002; Weston y Millward, 1992). Por el contrario, temas relacionados con sexualidad, política, religión o drogas no provocan conflictos con frecuencia. Como señalábamos algunas líneas atrás, algunos temas surgen como fuente de conflicto a medida que chicos y chicas van cumpliendo años -caso por ejemplo de la elección profesional o el uso del dinero-, sin embargo, determinados temas parecen ser motivo de discusión prácticamente durante toda la adolescencia (Smetana, 1989).

Según algunos estudios, adultos y adolescentes tienen visiones algo distintas de la realidad familiar (Barnes y Olson, 1985; Honess, Charman, Zani, Cicognani. Xerri, Jacdson y Bosma, 1997. Padres, y especialmente madres, perciben las interacciones de forma más positiva y optimista, tienden a infravalorar la tasa de conflictos y señalan más calidez y afecto de lo que indican sus hijos e hijas. Éstos por el contrario, parecen sobreestimar las diferencias existentes en el hogar, señalan más conflictos con sus padres y madres, y niveles más bajos de intimidad en la relación con ellos (Laursen, Coy y Collins, 1998; Noller y Callan, 1986; 1988; Smetana, 1989; Silverberg y Steinberg, 1990). 
Este trabajo de investigación pretende cubrir tres objetivos. En primer lugar, conocer la evolución que siguen los conflictos familiares a lo largo de la adolescencia, y hacerlo no sólo desde el punto de vista de los chicos y chicas, sino también de sus madres. Por otro lado, analizar las diferencias de género entre los adolescentes respecto a la frecuencia de los conflictos y a los temas concretos sobre los que discuten en casa. Finalmente, nuestro tercer objetivo es averiguar si realmente existen diferencias entre ambas perspectivas a la hora de percibir los conflictos familiares. Para responder de forma adecuada a ambas cuestiones hemos optado por un diseño longitudinal, el único que permite dar cuenta de los cambios que a nivel individual, se producen en nuestras vidas con el paso de los años. Además de estas dos grandes cuestiones, a lo largo del trabajo prestaremos especial atención a las diferencias entre chicos y chicas, para conocer así si existen discrepancias en la frecuencia con la que discuten con sus madres o en los temas que provocan conflictos.

\section{MÉTODO}

\section{Participantes}

\section{Adolescentes}

Este trabajo supone el seguimiento longitudinal de un grupo de chicos y chicas a lo largo de su adolescencia. Parte de una investigación previa en la que a través de un diseño transversal analizamos los cambios que se producían en la dinámica familiar coincidiendo con la adolescencia de hijas e hijos (Oliva y Parra, 2001; Parra y Oliva, 2002). En esta investigación la muestra estuvo compuesta por 513 adolescentes de edades comprendidas entre los 12 y los 19 años y pertenecientes a 10 centros educativos diferentes de Sevilla y su 
provincia. La elección de los colegios e institutos donde reclutamos a los adolescentes se realizó teniendo en cuenta criterios como su pertenencia al mundo rural o urbano, su titularidad -pública o privada concertada- y el nivel socioeconómico de las familias. Así intentamos que estuvieran representadas las diversas realidades de nuestro contexto.

La segunda fase de la investigación consistió en el seguimiento de los chicos y chicas que en el estudio anterior se encontraban en la adolescencia inicial, entre los 12 y los 14 años -media 13.11 y desviación tipo .44-. Este seguimiento se realizó durante más de cinco años, hasta que cumplieron los 18 o 19 años. Así, estos jóvenes completaron los instrumentos de evaluación en su adolescencia inicial, media y tardía, denominados Tiempo 1-T1-, Tiempo 2 -T2- y Tiempo 3-T3- respectivamente. La muestra final estuvo compuesta por 101 adolescentes, 38 chicos y 63 chicas, $74.3 \%$ de ellos pertenecientes al mundo urbano y $25.7 \%$ al rural. Las edades medias en la adolescencia media y tardía fueron 15.38 -desviación tipo .56- y 17.85 -desviación tipo .52- respectivamente.

Para identificar las posibles diferencias entre los jóvenes que continuaron en la investigación y aquellos que no lo hicieron, realizamos el Análisis de casos perdidos. Nuestros resultados indican que entre los sujetos que continuaron en la investigación hay algo más de chicas que de chicos $-\chi^{2}=4.05, p<.05-$, y menos hijos de padres de nivel educativo - profesional bajo $-\chi^{2}=6.52, p<.05-$. No obstante, son semejantes en cuanto a su hábitat -rural vs. urbano- y al tipo de centro educativo al que asisten -público vs. privado-. Tampoco son diferentes en el grado de afecto percibido en el hogar, aunque los que continuaron percibían algo más de control que los que no lo hicieron $-F(1,134)=3.89$, $p=.051-$.

\section{Madres}


Para tener una visión más completa de la dinámica familiar, en el Tiempo 2 de la investigación longitudinal decidimos entrevistar a los padres y madres de los adolescentes. Conviene señalar que los padres que quisieron colaborar fueron tan escasos -14 en T2 y 7 en T3-, que decidimos prescindir de ellos y utilizar sólo las entrevistas de las madres. No obstante, hubo algún caso en el que contamos con la entrevista del padre al ser él el cuidador principal. Así, en el Tiempo 2 la muestra estuvo compuesta por 69 sujetos -66 madres y 3 padres-, y en el Tiempo 3 por 49 madres y un padre. La edad de las madres osciló entre los 32 y los 56 años, con una media de 44.02 -desviación tipo 5.47-. El 43.7\% son amas de casa, y en cuanto a su nivel educativo, la mayoría-57\%- no tiene estudios o sólo tiene estudios primarios, el $17 \%$ tiene estudios medios y el $26 \%$ estudios universitarios.

Para saber si existían características diferenciales entre los adolescentes cuyas madres participaron en el estudio y aquellos cuyas madres no quisieron colaborar, realizamos un análisis comparativo. Los resultados indican que ambos grupos son semejantes en todas las variables. Por otro lado, el análisis de los sujetos perdidos no identificó diferencias significativas entre las madres que sólo participaron en T2 y aquellas que también lo hicieron en T3. Por ejemplo, unas y otras presentan niveles semejantes de conflictos con sus hijos e hijas $-\mathrm{F}(1,66)=.00$, p=n.s.-. El nivel educativo tampoco marcó diferencias entre las madres que siguieron y las que no $-\chi^{2}=3.90$, $p=$ n.s.- .

\section{Instrumentos}

\section{Datos de identificación}

Chicos y chicas respondieron a una serie de cuestiones demográficas tales como su edad y sexo, el centro educativo al que pertenecían y el curso en el que estaban. También 
describieron el nivel de estudios y la profesión de su padre y de su madre. Por otro lado, sus madres debían indicar su nivel de estudios alcanzado, edad y profesión.

\section{Conflictos familiares}

Es una escala de 14 ítems, creada ad hoc para esta investigación (Parra y Oliva, 2002), que evalúa la frecuencia de aparición de discusiones familiares sobre diversos temas -hora de volver a casa, amistades, drogas, política o religión, etc.- Alfas de Cronbach para este estudio T1 / T2 / T3 $\square=.81 / .62 / .71$. Los sujetos deben responder en una escala likert de 1 a 4 donde 1 implica no tener ninguna discusión y 4 tener discusiones con mucha frecuencia. En esta escala no se diferencian las discusiones del adolescente con madre y padre, sino que se plantean las discusiones con ambos conjuntamente. Así, este instrumento fue completado por los chicos y las chicas en referencia a los conflictos con sus padres en general, y por las madres en referencia a los conflictos con sus hijos e hijas adolescentes.

\section{Procedimiento}

\section{$\underline{\text { Adolescentes }}$}

La primera recogida de datos -T1- se realizó durante el curso académico 19981999. La segunda -T2- tuvo lugar en el 2000-2001, y la tercera -T3- en el 2002-2003. El primer paso fue seleccionar los centros educativos y ponernos en contacto con su equipo directivo para explicarles la investigación y solicitar su colaboración. Una vez que aceptaron participar con nosotros, seleccionamos las aulas en las que recogeríamos los datos. A continuación enviamos una carta a los padres y madres de los adolescentes solicitando su permiso para que colaboraran en la investigación. Una vez obtenido el permiso, pasamos a aplicar los cuestionarios de forma anónima y colectiva. Para facilitar el seguimiento posterior, cada uno de los participantes tenía un identificador numérico que equivalía a su nombre y apellidos, y que sólo los investigadores conocíamos. 
En la tercera recogida de datos, T3, algunos adolescentes no estaban escolarizados o lo estaban en centros distintos a los de T1. En estos casos, una vez que contactamos con ellos y aceptaban seguir colaborando en la investigación, concertamos una cita para que completaran el cuestionario en el seminario del Dpto. de Psicología Evolutiva y de la Educación de la Universidad de Sevilla.

\section{$\underline{\text { Madres }}$}

El primer paso consistió en contactar telefónicamente con las madres y/o los padres, explicarles los objetivos de la investigación y solicitar su colaboración. Una vez que aceptaban colaborar con nosotros, se fijaba la fecha de la entrevista y el lugar. La mayoría de dichas entrevistas se realizaron en el hogar familiar, aunque alguna madre prefirió para ello el seminario del Dpto. de Psicología Evolutiva y de la Educación de la Universidad de Sevilla. En ambos casos, alguno de los miembros del equipo de investigación realizaba la entrevista.

\section{RESULTADOS}

Los resultados que presentamos a continuación están divididos en función de las dos grandes cuestiones que guían nuestro trabajo de investigación. En primer lugar, analizar la evolución de la frecuencia de los conflictos a lo largo de la adolescencia desde la perspectiva de madres y adolescentes; y en segundo lugar, conocer si existen diferencias entre ambas.

Para responder a la primera cuestión vamos a presentar los resultados diferenciando la estabilidad absoluta y relativa de las variables. La estabilidad absoluta de una variable hace referencia a su consistencia a lo largo del tiempo, y supone analizar cómo se comporta su valor promedio en los distintos tiempos de medida. No obstante, y al basarse en puntuaciones medias, no nos informa de los cambios individuales y no da cuenta, en el caso 
de que las haya, de trayectorias diferentes seguidas por grupos de sujetos. Para profundizar en este aspecto presentaremos resultados sobre su estabilidad relativa. La estabilidad relativa permite conocer la consistencia de la posición de los sujetos respecto a su grupo de referencia a través del tiempo, y determinar si se sitúan de forma similar en los diferentes momentos de observación comparados con su grupo. El procedimiento más utilizado para medir la estabilidad relativa de las variables es el que se basa en los coeficientes de correlación entre los diferentes tiempos de medida (Alder y Scher, 1994).

Además de la estabilidad absoluta y relativa, para profundizar en la evolución seguida por grupos de sujetos hemos llevado a cabo análisis de conglomerados. Con este procedimiento hemos pretendido identificar grupos de adolescentes semejantes en función de su trayectoria en la frecuencia de conflictos. Así, esta información nos indica si las trayectorias observadas a través de la estabilidad absoluta son comunes a todos los sujetos o podemos identificar grupos distintos, lo que sin duda, ayuda a complementar los datos procedentes del análisis de la estabilidad relativa. Para llevar a cabo el análisis de conglomerados utilizamos dos procedimientos sucesivos. En primer lugar realizamos un análisis de conglomerados con $K$ medias, que nos permite reducir en número de sujetos inicial a sólo 10 grupos -número que elegimos aleatoriamente- en función de las semejanzas de las trayectorias seguidas a lo largo de la adolescencia. Una vez reducido el número de casos, utilizamos el procedimiento de conglomerados jerárquicos para constituir el número final de grupos que consideramos homogéneo respecto a las trayectorias de los sujetos que lo componen. Es importante tener en cuenta que el análisis de conglomerados jerárquicos genera distintas agrupaciones, por lo que seremos nosotros los que tras la valoración de las diferentes soluciones, elijamos la que consideremos más consistente y la que agrupa a los sujetos de forma más natural. 
1. Evolución de la frecuencia de los conflictos a lo largo de la adolescencia desde la perspectiva de madres y adolescentes

\section{a. Adolescentes}

El instrumento de evaluación consistía en 14 temas ante los que los adolescentes tenían que indicar la frecuencia con la que provocaban discusiones Para obtener una única medida de la frecuencia general de los conflictos en cada uno de los tiempos de medida generamos una nueva variable a través de las medias de las respuestas de los sujetos ante los 14 temas conflictivos.

\section{i. Estabilidad absoluta}

Cuando analizamos la evolución de la frecuencia de los conflictos -figura 1-, los datos indican una diferencia significativa a lo largo de los años -Traza de Pillai $F(2,98)=3.15, \mathrm{p}=<.05-$ que implica un decremento en las discusiones especialmente entre la adolescencia media y tardía.

\section{Insertar Figura 1 aproximadamente aquí}

Al analizar por separado las tendencias de los chicos y las chicas, observamos que existen diferencias significativas sólo en el caso de las primeras -Traza de Pillai, F(2, $98)=3.50, p=<.05-$ que perciben un decremento en la frecuencia de los conflictos especialmente entre la adolescencia media y tardía. Los chicos no aprecian cambios significativos a lo largo de los años -Traza de Pillai, $F(2,98)=1.05$, $p=$ n.s.-.

Por otro lado, los datos indican que aunque los chicos afirman tener discusiones algo más frecuentes que las chicas, estas diferencias no son significativas en ningún tramo de la adolescencia. En cualquier caso, y como veremos a continuación, cuando existen 
diferencias en la frecuencia de conflictos percibida por unos y otras respecto a temas concretos, son los primeros los que afirman tener más discusiones. Sólo existe una excepción: la hora de volver a casa, ya que sobre este tema son las adolescentes quienes tienen más riñas.

\section{Trayectorias evolutivas}

Tras la realización de un análisis de conglomerados de K-medias redujimos todos los sujetos a sólo 10 grupos en función de la semejanza de sus puntuaciones respecto a la frecuencia de conflictos con sus madres y padres. A continuación realizamos un análisis de conglomerados jerárquicos, a través del cual decidimos optar por la existencia de tres grupos. En la figura 2 aparecen las trayectorias de los tres grupos generados. Como vemos en dicha figura, existen diferencias significativas entre ellos. El primero y más numeroso manifiesta una tendencia estable a lo largo de los años, mientras que el segundo presenta un decremento importante en la frecuencia de conflictos entre la adolescencia inicial y media, y el tercero un ligero incremento en este mismo período.

Insertar Figura 2 aproximadamente aquí

Es interesante señalar que los resultados del análisis de conglomerados matizan los obtenidos a través del análisis de medidas repetidas, y apuntan a que la elevada estabilidad absoluta no es general para todos los sujetos, sino que existen algunos individuos que presentan cambios significativos en la frecuencia de los conflictos con sus madres y padres. Así, habría que destacar el importante descenso en la conflictividad que se observa en algunos sujetos que presentaban niveles muy altos de discusiones al inicio de la adolescencia. 
Cuando relacionamos los grupos anteriores con el sexo adolescente los resultados indican que existen claras diferencias de género $-\chi^{2}=8,107, \mathrm{p}=<.01-$. Mientras que en el primer grupo hay una frecuencia de chicas mayor de lo esperable por azar, en el grupo 2, en el que se observa una acusada disminución de las discusiones, existen más chicos. En el tercer grupo, chicos y chicas están igualmente representados.

\section{ii. Estabilidad relativa}

Nuestros datos indican una estabilidad prácticamente idéntica para el caso de las chicas y de los chicos. Además, tanto para unos como para otras la mayor estabilidad aparece entre la adolescencia media y tardía -ver tabla I-. Esta mayor consistencia indica que las posiciones relativas respecto a sus grupos de referencia se mantienen más estables en los últimos años de la adolescencia que en los primeros.

Insertar tabla I aproximadamente aquí

Tomados conjuntamente, los datos sobre la estabilidad absoluta y relativa indican que entre la adolescencia inicial y media no existen cambios significativos en la frecuencia promedio de los conflictos porque hay jóvenes que perciben un aumento en las discusiones mientras que otros señalan un decremento que compensa el incremento anterior. Si recordamos los grupos obtenidos tras el análisis de conglomerados, entre la adolescencia inicial y media, además de un grupo muy numeroso que se mantenía constante respecto a la frecuencia de los conflictos, había un grupo, formado sobre todo por chicos, que disminuía claramente la frecuencia de conflictos mientras que un tercero la aumentaba ligeramente.

Entre la adolescencia media y tardía, independientemente de que las medias se mantengan -caso de los chicos- o que disminuyan ligeramente -caso de las chicas- los 
adolescentes mantienen su posición relativa respecto al grupo de referencia. En otras palabras, los jóvenes no advierten cambios entre la adolescencia media y tardía en la frecuencia de los conflictos, y aquellos que hablaban de más discusiones en el primer momento también siguen considerando que tienen más discusiones en el segundo. En el caso de las adolescentes, aunque describen una ligera disminución en los conflictos, esta disminución es general para todas, para aquellas que percibían más conflictos y para las que percibían menos, manteniéndose sus posiciones relativas respecto al grupo. Por lo tanto, parece que las fluctuaciones mayores en la conflictividad, tanto en lo referente a estabilidad absoluta como relativa, tienen lugar entre la adolescencia inicial y media. En cualquier caso, conviene destacar que el grupo más numeroso de la muestra mantiene una frecuencia de conflictos estable y relativamente baja a lo largo de los años. En una escala de 1 a 4, donde 1 significa no tener ninguna discusión y 4 tener muchas, se sitúan alrededor de 1,5.

Cuando analizamos la frecuencia de discusiones teniendo en cuenta los temas concretos origen de conflicto y diferenciando entre chicos y chicas, aparecen datos interesantes. Cuando existen diferencias en la tasa de conflictos entre los y las adolescentes, en general, ellos perciben más discusiones que ellas. Este es el caso del empleo del tiempo libre $-\mathrm{F}(1,91)=5.26, \mathrm{p}=<.05-$, las horas de estudio y las notas $-\mathrm{F}(1,91)=8.72, \mathrm{p}=<.01-$, el consumo de tabaco y alcohol $-\mathrm{F}(1,91)=3.79, \mathrm{p}=<.05-\mathrm{y}$ otras drogas $-\mathrm{F}(1,91)=15.39$, $\mathrm{p}=<.01$ - o la carrera profesional a seguir $-\mathrm{F}(1,91)=8.42, \mathrm{p}=<.01-$. Sólo hay un tema en el que las chicas afirman tener más discusiones que sus compañeros varones: la hora de llegar a casa $-\mathrm{F}(1,91)=5.51, \mathrm{p}=<.05-$. Un tema que parece ser especialmente conflictivo en la adolescencia media.

En el caso de los chicos, el momento en el que se producen más discusiones es la adolescencia inicial. A partir de ahí hay una disminución en los conflictos especialmente 
sobre aspectos cotidianos como la forma de vestir o arreglarse $-\mathrm{F}(2,91)=3.10, \mathrm{p}=<.05-$, sus ligues $-\mathrm{F}(2,91)=3.43, \mathrm{p}=<.05-\mathrm{y}$ su conducta sexual $-\mathrm{F}(2,90)=10.39, \mathrm{p}=<.01-$.

Respecto a los temas que provocan discusiones familiares con mayor frecuencia, existe bastante consenso entre chicos y chicas. En general para todos, y en todos los momentos de la adolescencia, las tareas del hogar, el empleo del dinero, la hora de recogida, el tiempo de estudio o la forma de vestir son los asuntos que provocan más discusiones. Por el contrario, los que provocan menos riñas son la política o la religión, lo relacionado con su conducta sexual o ligues, y las drogas.

b. Madres

i. Estabilidad absoluta y relativa

En los siguientes párrafos analizaremos los cambios en la percepción de las madres respecto a los conflictos con sus hijos e hijas entre la adolescencia media y tardía. Al igual que hicimos en el apartado anterior, comenzaremos por la estabilidad absoluta de las variables para tratar posteriormente la relativa. Al tener sólo dos medidas de las variables, recogidas durante la adolescencia media -T2- y tardía -T3-, en los análisis estadísticos utilizaremos la prueba $t$ de comparación de medias para muestras relacionadas.

En primer lugar, podemos señalar que las madres no afirman tener muchos conflictos con sus hijos e hijas durante la adolescencia, ya que en una escala de 1 -ninguna discusión- a 4 -muchas discusiones-, la media no llega a alcanzar el 2 -algunas discusiones-. La tabla 2 muestra una estabilidad relativa media en la frecuencia de los conflictos, lo que significa que entre la adolescencia media y tardía se producen ligeros cambios en las posiciones relativas de las madres respecto a dichas variables.

Insertar Tabla II aproximadamente aquí 
Teniendo en cuenta la evolución sobre temas concretos, nuestros resultados indican que para las madres, a medida que transcurren los años, se produce un decremento en los conflictos sobre temas cotidianos como la hora de llegar a casa $-\mathrm{t}(48)=2.76, \mathrm{p}=<.01-$, la forma de gastar el dinero $-\mathrm{t}(48)=2.73, \mathrm{p}=<.01-$, el tiempo de estudio y las calificaciones académicas $-\mathrm{t}(48)=2.66, \mathrm{p}=<.01-$. Temas que por otro lado, eran de los que provocaban discusiones con mayor frecuencia en la adolescencia media. Como dijimos en líneas anteriores, en general, las madres no perciben conflictos muy frecuentes con sus hijos e hijas, de hecho, el único tema que sigue provocando discusiones con cierta frecuencia en la adolescencia tardía es el referido a la realización de las tareas del hogar.

2. Diferencias en la percepción de madres y adolescentes.

En primer lugar, y como muestra la tabla III, los hijos e hijas perciben mayor tasa de conflictos en los dos momentos de la adolescencia observados.

\section{Insertar Tabla III aproximadamente aquí}

Cuando analizamos los temas concretos en los que se producen discrepancias entre madres y adolescentes, los datos indican que existen algunas diferencias entre la adolescencia media y tardía. Si bien las discusiones sobre los amigos y amigas son percibidas con mayor frecuencia por los y las adolescentes tanto en la adolescencia media $\mathrm{t}(68)=-2.00, \mathrm{p}=<.05-$ como en la tardía $-\mathrm{t}(48)=-2.07, \mathrm{p}=<.05-$. En el primero de estos momentos también existen diferencias entre madres y adolescentes en temas como la conducta sexual de chicas y chicos $-\mathrm{t}(68)=-2.05, \mathrm{p}=-<.05-, \mathrm{o}$ los sitios a los que van cuando salen $-\mathrm{t}(68)=-3.36, \mathrm{p}=<.05-$. Por otro lado, en la adolescencia tardía las diferencias más importantes entre madres y adolescentes se refieren al tiempo de estudio y a las 
calificaciones escolares $-\mathrm{t}(48)=-2.96, \mathrm{p}=<.01-$, al empleo del dinero $-\mathrm{t}(48)=-3.18, \mathrm{p}=<.01-$ y a temas menos cotidianos como la política y la religión $-\mathrm{t}(48)=-2.55, \mathrm{p}=<.05$-. En cualquier caso, es importante recordar que chicas y chicos perciben mayor frecuencia de discusiones respecto a todos los temas anteriores que sus madres.

\section{DISCUSIÓN}

Los resultados de nuestro estudio subrayan la continuidad de los conflictos familiares a lo largo de la adolescencia, ya que para la mayoría de los sujetos de la muestra no se producen cambios significativos en el número de discusiones que mantienen con sus madres y padres. No obstante, también apuntan a una ligera disminución con los años. Este resultado coincide con el obtenido en el metaanálisis de Laursen y Collins (1998). Aunque al analizar las trayectorias individuales descubrimos que la disminución no es generalizada, sino que ocurre sólo para las chicas entre la adolescencia media y la tardía, y para el grupo de chicos que más conflictos presentaba en la adolescencia inicial.

Por otro lado, hemos encontrado que, aunque no aparecen grandes cambios en función de los temas concretos objeto de discusión, con la edad aumentan los conflictos sobre la hora de llegar a casa, especialmente para las chicas. En el caso de los chicos con los años disminuyen las discusiones sobre la forma de vestir, los ligues, la política y la religión.

La adolescencia es un momento de transición en el sistema familiar que exige de sus miembros un esfuerzo de adaptación, no obstante, las nuevas formas de funcionamiento que se adquieran en este momento dependerán en parte de las que ya existían en años anteriores, por lo que la trayectoria seguida por el sistema familiar tenderá a la coherencia y a la continuidad (Lewis, 1995; 1997; Granic, Dishion y Hollenstein, 2003). En nuestra 
investigación esta coherencia se refleja en que las posiciones relativas de los sujetos con respecto a su grupo de referencia tienden a mantenerse constantes en los diferentes momentos de observación. Es decir, independientemente de cuál sea la tendencia general puesta de manifiesto a través de las medias, los jóvenes que afirmaban tener más conflictos, por ejemplo en la adolescencia tardía, son los que tendían a ocupar los puestos superiores también en los años intermedios.

La adolescencia es un momento de transición -o de transiciones, como señalan Graber, Brooks-Gunn y Petersen (1996) o Lerner et al., (1996)- en la vida de chicos y chicas. Un periodo plagado de transformaciones y mudanzas que van a resultar necesarias para situar a los y las jóvenes a las puertas del mundo adulto. En este sentido, no es extraño que la adolescencia, y especialmente sus primeros momentos sean los más conflictivos en la relación entre padres e hijos. En la medida que unos y otros sean capaces de alcanzar un nuevo equilibrio, las relaciones se normalizarán y las riñas y discusiones tenderán a bajar.

A pesar de lo comentado hasta ahora sobre el incremento de la frecuencia de los conflictos a lo largo de la adolescencia, nuestros datos presentan una imagen bastante normalizada de este momento, y coinciden con otros muchos trabajos según los cuales menos del 10\% de las familias tienen serias dificultades en este momento (Dekovic, 1999; Motrico, Fuentes y Bersabé, 2001; Parra, Oliva y Nobes, 2000; Weston y Millward, 1992). De hecho, la mayoría de nuestros adolescentes afirma discutir poco con sus madres. Como señalan otros trabajos, la mayoría de las familias que manifiestan graves problemas ya los presentaban durante la infancia (Collins, 1997; Holmbeck, Paikoff y Brooks-Gunn, 1995; Rueter y Conger, 1995; Steinberg, 1990), por lo que aunque las riñas y discusiones son una realidad normativa durante la transición familiar de la adolescencia, los conflictos graves no lo son (Steinberg y Silk, 2002). 
Uno de los objetivos de nuestro trabajo era detectar la existencia de diferencias de género entre los adolescentes, e intentar explicarlas. Según nuestros datos, en general, chicos y chicas muestran un nivel de conflictos semejante con sus madres y padres. Sin embargo, cuando analizamos los temas concretos, se presenta una realidad bastante menos simple, de la que se desprende que chicos y chicas continúan percibiendo un trato diferente en el hogar. Un ejemplo: ellos tienen más conflictos sobre temas académicos, incluyendo la carrera o profesión que quieren elegir, sobre su uso del tiempo libre, sobre el tabaco, el alcohol y otras drogas. El único tema que genera más discusión entre las jóvenes es el de la hora de llegar a casa, un tema que probablemente ellos decidan de forma más independiente que ellas.

Siguiendo con los temas que generan conflictos con mayor y menor frecuencia, nuestros resultados coinciden plenamente con los procedentes de trabajos clásicos de investigación según los cuales, las discusiones más numerosas son las referidas a asuntos de la vida cotidiana. Temas escolares como el tiempo que los jóvenes dedican a estudiar y las notas que obtienen, y cotidianos como las tareas del hogar, la forma de vestir o el empleo del dinero. ¿Por qué son más frecuentes las discusiones sobre estos temas de la vida cotidiana? Según Judith Smetana (1988; 1989; Smetana y Asquith, 1994) muchos de los conflictos que surgen en el hogar no son sólo fruto de desacuerdos, sino de una forma distinta de razonar sobre la legitimidad de la autoridad parental. Así por ejemplo, mantener limpia la habitación es considerado por la mayoría de los jóvenes como algo perteneciente a su esfera privada, mientras que sus madres y padres suelen hacer una interpretación más convencional, que apela a la autoridad, la responsabilidad, las normas o las costumbres.

Otra posible causa de los desacuerdos puede residir en las distintas expectativas que tienen unos y otros respecto al momento que consideran más apropiado para iniciar algunos 
comportamientos tales como ir a discotecas o pasar un fin de semana fuera de casa. Algunos estudios han encontrado una mayor precocidad en las expectativas de los adolescentes que de sus padres y madres (Dekovic, Noom y Meeus, 1997; Casco y Oliva, 2005), lo que sin duda provocará discusiones y conflictos.

Una tercera explicación ante la mayor tasa de conflictos sobre temas cotidianos parte de la influencia que ejerce el grupo de iguales, y que parece afectar mucho más a este tipo de comportamientos que a aspectos relacionados con la moral, la religión o la orientación vocacional, donde padres y madres ejercen una influencia mayor (Brown, 1990). Así, tiene sentido pensar que los conflictos serán más frecuentes en aquellos temas donde los adultos pierden poder de influencia, y donde probablemente, no siempre estén de acuerdo con la opinión del grupo de amigos de sus hijos e hijas.

Uno de los aspectos más interesantes de nuestro trabajo, y que coincide con los hallazgos de diferentes autores, es que madres y adolescentes se acercan a la realidad familiar con lentes de distinta tonalidad, unas lentes que ofrecen a las primeras un matiz más cálido, y a los segundos uno más oscuro y gris (Laursen, Coy y Collins, 1998; Noller y Callan, 1986; 1988; Silverberg y Steinberg, 1990; Smetana, 1989). Concretamente, las madres de nuestra muestra describen menos conflictos y de menor intensidad de lo que dicen sus hijos e hijas. Según la hipótesis de la "Apuesta generacional" -“Generational stake"- propuesta por Bengtson (1971) adultos y adolescentes tenderían a destacar aspectos distintos de la relación. Padres y madres han invertido mucho tiempo y esfuerzo en formar un hogar y en criar a hijos e hijas, por lo que tienen que esforzarse en ver la cara amable de la relación con ellos. Por el contrario, los adolescentes se encuentran en un momento en el que tienen que reafirmar su autonomía e independencia, lo que les empuja a centrarse en los aspectos más negativos de la relación. En otras palabras, percibir las relaciones con los 
hijos de forma muy problemática generaría tal frustración en los adultos, que tenderían a maquillar la realidad o, al menos, a ver su lado más positivo. Sin embargo, lo adaptativo para el adolescente es destacar las dificultades que tiene en las relaciones con sus padres y así reafirmarse en su deseo y necesidad de independencia (Hones et al., 1997). Una hipótesis complementaria a la que acabamos de presentar destaca el papel de la deseabilidad social como factor explicativo de la visión más negativa que los adolescentes presentan de la dinámica familiar (Hartos y Power, 2000). Y es que si bien para padres y madres lo deseable es dar una imagen positiva de las relaciones en el hogar, para hijos e hijas lo deseable puede ser justo lo contrario, y la actitud del joven rebelde le sirve para reafirmar su autonomía e independencia, algo especialmente importante en este momento evolutivo.

En este sentido, en un conocido trabajo, Gonzales, Cauce y Mason (1996) concluyen que la opinión de los adolescentes es la que coincide en mayor medida con los registros de observadores externos, siendo la perspectiva más fiable. A nuestro juicio, el que esto sea así no resta importancia a la información aportada por las madres, por el contrario, nos permite conocer la vivencia de los otros protagonistas de la historia, unos protagonistas que están en un momento vital distinto y que están inclinados a ver una realidad familiar algo más dulce.

Este trabajo tiene algunas limitaciones. Quizás una de las más importantes es el escaso número de madres que participaron. Contar con un número mayor nos hubiera permitido comparar por ejemplo su percepción en función del sexo de los hijos, analizando si las madres de las chicas ven las relaciones de forma distinta a las madres de los chicos. Igualmente, hubiera sido muy interesante contrastar su visión con la de los padres. Otra de las limitaciones de nuestro trabajo tiene que ver con la utilización exclusiva de 
cuestionarios como método de recogida de información. Ambos aspectos no restan valor a nuestros resultados. Simplemente sugieren tomarlos con cierta prudencia a la hora de considerar su posible generalización.

Este trabajo aporta algunas ideas interesantes con claras implicaciones prácticas. Entre ellas nos gustaría destacar la continuidad y estabilidad que parece dominar en esta etapa. Así, las trayectorias de los adolescentes parecen ser bastante coherentes, y en general, aquellos chicos y chicas que se sitúan en los niveles superiores en cuanto a la frecuencia de los conflictos con sus padres, tienden a ser los que ocupan las posiciones superiores en años posteriores. En este sentido, aparece especialmente importante detectar e intervenir de forma temprana sobre las dificultades que puedan aparecer en el desarrollo de los jóvenes y en el funcionamiento de sus familias, comenzando en los primeros años de la adolescencia o incluso en los últimos de la infancia.

Por otro lado, nuestros resultados han puesto de manifiesto que madres y adolescentes tienen perspectivas algo distintas de la realidad familiar. En este sentido, padres y madres deben ser conscientes de los procesos de cambio que están experimentando sus hijos e hijas, y de la forma en que van a afectar a la realidad familiar, teniendo presente que deberán ajustar sus prácticas a las nuevas necesidades del adolescente y a su creciente autonomía (Collins y Laursen, 2004). Igualmente, deben tener en cuenta que ellos también están experimentando cambios, lo que puede hacerlos especialmente sensibles a las dificultades en las interacciones con sus hijos. 


\section{REFERENCIAS BIBLIOGRÁFICAS}

Alder, A. G., y Scher, S. J. (1994). Using growth curve analyses to assess personality change and stability in adulthood. En T. F. Heatherton, y Weinberger, J. L. (Ed.), Can personality change? Washington, DC: American Psychological Association.

Arnett, J. J. (1999). Adolescent Storm and Stress, Reconsidered. American Psychologist, 54(5), 317-326.

Barnes, H. L., y Olson, D. H. (1985). Parent-adolescent communication and the circumplex model. Child Development, 56, 438-447.

Bengtson, V. L., y Kuypers, J. A. (1971). Generational differences and the developmental stake. Aging and human development, 2, 249-260.

Bengtson, V. L., y Troll, L. (1978). Youth and their parents: Feedback and intergenerational influence in socialization. En R. M. Lerner, y Spanier, G. B. (Ed.), Child influences on marital and family interaction: a life-span perspective (pp. 215236). Nueva York: Academic.

Blos, P. (1979). Modifications in the classical psychoanalytic model of adolescence. Adolescent psychiatry, 7, 6-25.

Bosma, H. A., Jackson, S. E., Zusling, D. H., Zani, B., Cicognani, E., Xerri, L. M., Honess, T. M., y Charman, L. (1996). Who has the final say? Decision on adolescent behaviour within the family. Journal of Adolescence, 19, 277-291.

Brown, B. (1990). Peer groups. En S. Feldman, y Elliot, G. (Ed.), At the threshold: the developing adolescent (pp. 171-196). Cambridge MA: Harvard University Press.

Casco, F. J., y Oliva, A. (2005). Valores y expectativas sobre la adolescencia. Discrepancias entre padres, profesores, mayores y adolescentes. Infancia $y$ Aprendizaje, 28, 209-220.

Collins, W. A. (1997). Relationships and development during adolescence. Interpersonal adaptation to individual change. Personal Relationships, 4, 1-14. 
Collins, W. A., y Laursen, B. (2004). Parent-adolescent relationships and influences. En R. M. Lerner, y Steinberg, L. (Ed.), Handbook of adolescent psychology. (pp. 331361). Nueva Jersey.: Willey.

Conger, R. D., y Ge, X. (1999). Conflict and cohesion in parent-adolescent relations: changes in emotional expression from early to mid-adolescence. En M. Cox, y Brooks-Gunn, J. (Ed.), Conflict and cohesion in families: causes and consequences (pp. 185-206). Mahwah, Nueva Jersey: Lawrence Erlbaum Associates.

Dekovic, M. (1999). Parent-adolescent conflict: possible determinants and consequences. International journal of behavioural development, 23, 977-1000.

Dekovic, M., Noom, M. J. y Meeus, W. (1997). Expectations regarding development during adolescence: Parental and adolescent perception. Journal of youth and adolescence, 26, 253-272.

Dolgin, K. G. (1996). Parents' disclosure of their own concerns to their adolescent children. Personal relationships, 3, 159-169.

Elzo, J., Orizo, F. A., González-Anleo, J., González, P., Laespada, M. T., y Salazar, L. (1999). Jóvenes españoles 1999. Madrid: Fundación Santa María.

Galambos, N. L., y Almeida, D. M. (1992). Does parent-adolescent conflict increase in early adolescence? Journal of Marriage and the Family, 54, 737-747.

Gonzales, N. A., Cauce, A. M., y Mason, C. A. (1996). Inter-observer agreement in the assessment of parental behaviour and parent-adolescent conflict: African-American mothers, daughters and independent observers. Child Development, 67, 1483-1498.

Graber, J. A., Brooks-Gunn, J., y Petersen, A. C. (1996). Adolescent transitions in context. En J. A. Graber, Brooks-Gunn, J., y Petersen, A. C. (Ed.), Transitions through adolescence: Interpersonal domains and context (pp. 369-383). Mahwah, Nueva Jersey: Erlbaum.

Granic, I., Dishion, T. J. y Hollenstein, T. (2003). The family ecology of adolescence: A dynamic systems perspective on normative development. En G. R. Adams, y Berzonsky, M. (Ed.), The Blackwell Handbook of Adolescence. Oxford, Reino Unido: Blackwell. 
Hartos, J. L., y Power, T. G. (2000). Association between mother and adolescent reports for assessing relations between parent-adolescent communication and adolescent adjustment. Journal of Youth and Adolescence, 29(4), 441-450.

Honess, T. M., Charman, E.A., Zani, B., Cicognani, E., Xerri, M. L., Jackson, A.E., y Bosma, H.A. (1997). Conflict between parents and adolescents: Variation by family constitution. British journal of Developmental Psychology, 15, 367-385.

Holmbeck, G. N., y Hill, J. P. (1991). Conflictive Engagement, Positive Affect and Menarche in Families with Seventh-Grade Girls. Child Development, 62, 10301048.

Holmbeck, G. N., Paikoff, R. L., y Brooks-Gunn, J. (1995). Parenting Adolescents (capítulo 4). En M. H. Bornstein (Ed.), Handbook of Parenting (Vol. 1, pp. 91-118). Nueva Jersey: Lawrence Erlbaun Associates.

Laursen, B., Coy, K. C., y Collins, W. A. (1998). Reconsidering Changes in Parent-Child Conflict across Adolescence: A Meta-Analysis. Child Development, 69(3), 817-832.

Lerner, R. M., Lerner, J. V., von Eye, A., Ostrum, C. W., Nitz, K., Talwar-Soni, R., y Tubman, J. (1996). Continuity and discontinuity across the transition of early adolescence: A developmental contextual perspective. En J. A. Graber, BrooksGunn, J., y Petersen, A. C. (Ed.), Transitions through adolescence: Interpersonal domains and context (pp. 3-22). Mahwah, Nueva Jersey: Erlbaum.

Lewis, M. D. (1995). Cognition-emotion feed-back and the self-organization of developmental paths. Human Development, 38, 71-102.

Lewis, M. D. (1997). Personality self-organization: Cascading constraints on cognitionemotion interaction. En A. Fogel, Lyra, M., y Valsiner, J. (Ed.), Dynamics and indeterminism in developmental and social processes (pp. 193-216). Mahwah Nueva Jersey: Erlbaum.

Montemayor, R. (1983). Parents and adolescents in conflict: All families some of the time and some families most of the time. Journal of Early Adolescence, 3, 83-103.

Motrico, E., Fuentes, M. J., y Bersabé R. (2001). Discrepancias en la percepción de los conflictos entre padres e hijos/as a lo largo de la adolescencia. Anales de Psicología, $17(1), 1-13$. 
Noller, P. (1994). Relationship with parents in Adolescence: Process and Outcomes. En R. Montemayor, Adams, G.R., y Gullota, T.P. (Ed.), Personal Relationship During Adolescence: Thousand Oaks, CA: SAGE.

Noller, P., y Callan, V. J. (1986). Adolescent and parent perceptions of family cohesion and adaptability. Journal of adolescence, 9, 97-106.

Noller, P., y Callan, V. J. (1988). Understanding parent-adolescent interaction: the perception of family members and outsiders. Developmental Psychology, 24(5), 707-714.

Paikoff, R. L., y Brooks-Gunn, J. (1991). Do parent-child relationships change during puberty? Psychological Bulletin, 110, 47-66.

Parra, A., y Oliva, A. (2002). Comunicación y conflicto familiar durante la adolescencia. Anales de psicología, 18, 215-231.

Parra, A., Oliva, A., y Nobes, G. (2000,). Parent-child communication and family conflict during adolescence: a cross-cultural study. Comunicación presentada en el VIIth biennial meeting de la European Association For Research in Adolescence. Jena, Alemania.

Rueter, M., y Conger, R. D. (1995). Antecedents of Parent-Adolescent Disagreements. Journal of Marriage y Family, 57(2), 435-449.

Silverberg, S. B., y Steinberg, L. (1990). Psychological Well-Being of parents With Early Adolescent Children. Developmental Psychology, 26(4), 658-656.

Smetana, J. A., P. (1994). Adolescents' and parents' conceptions of parental authority and personal autonomy. Child Development, 65, 1147-1162.

Smetana, J. G. (1988). Adolescents' and parents' conceptions of parental authority. Child Development, 59, 321-335.

Smetana, J. G. (1989). Adolescents' and Parents' Reasoning about family conflict. Child Development, 60, 1052 - 1067.

Steinberg, L. (1987). Impact of puberty on family relations: effects of pubertal status and pubertal timing. Developmental psychology, 23(3), 451-460.

Steinberg, L. (1988). Reciprocal relations between parent-child distance and pubertal maturation. Developmental psychology, 24(1), 122-128. 
Steinberg, L. (1990). Interdependence in the family: autonomy, conflict and harmony in the parent-adolescent relationship. En S. S. Feldman, y Elliott, G. L. (Ed.), At the threshold: the developing adolescent (pp. 255-276). Cambridge, MA: Harvard University Press.

Steinberg, L., y Silk, J. S. (2002). Parenting adolescents. En I. Bornstein (Ed.), Handbook of parenting (Vol. I. Children and parenting). Mahwah, Nueva Jersey: Lawrence Erlbaum Associates.

Weston, R., y Millward, C. (1992). Adolescent children and their parents. Family matters, 33, 36-39.

Weston, R., y Millward, C. (1992). Adolescent children and their parents. Family matters, 33, 36-39. 
Figura 1. Evolución de la frecuencia de los conflictos según la opinión de chicas y chicos

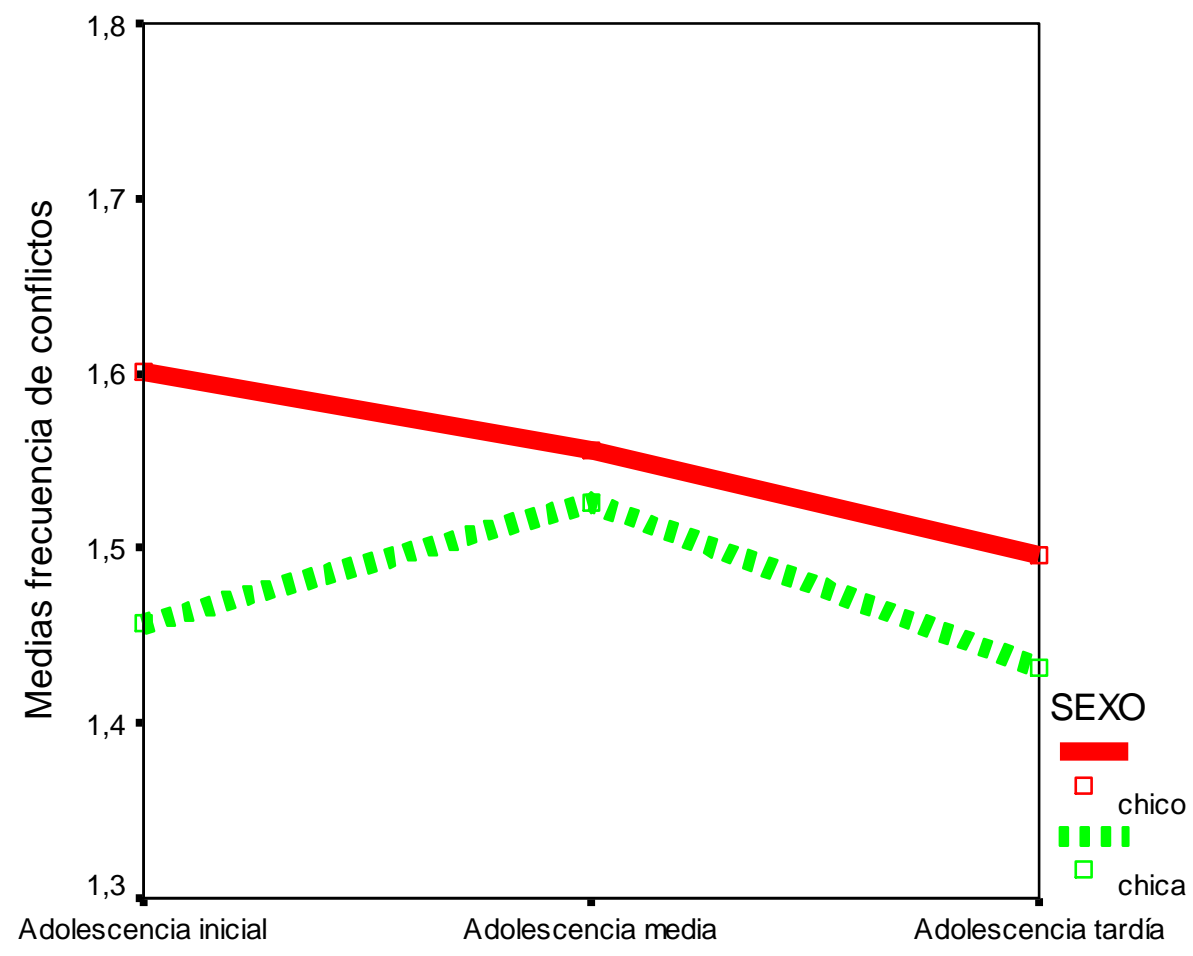


Figura 2. Trayectoria de los grupos obtenidos en función de la evolución de la frecuencia de los conflictos con padres y madres.

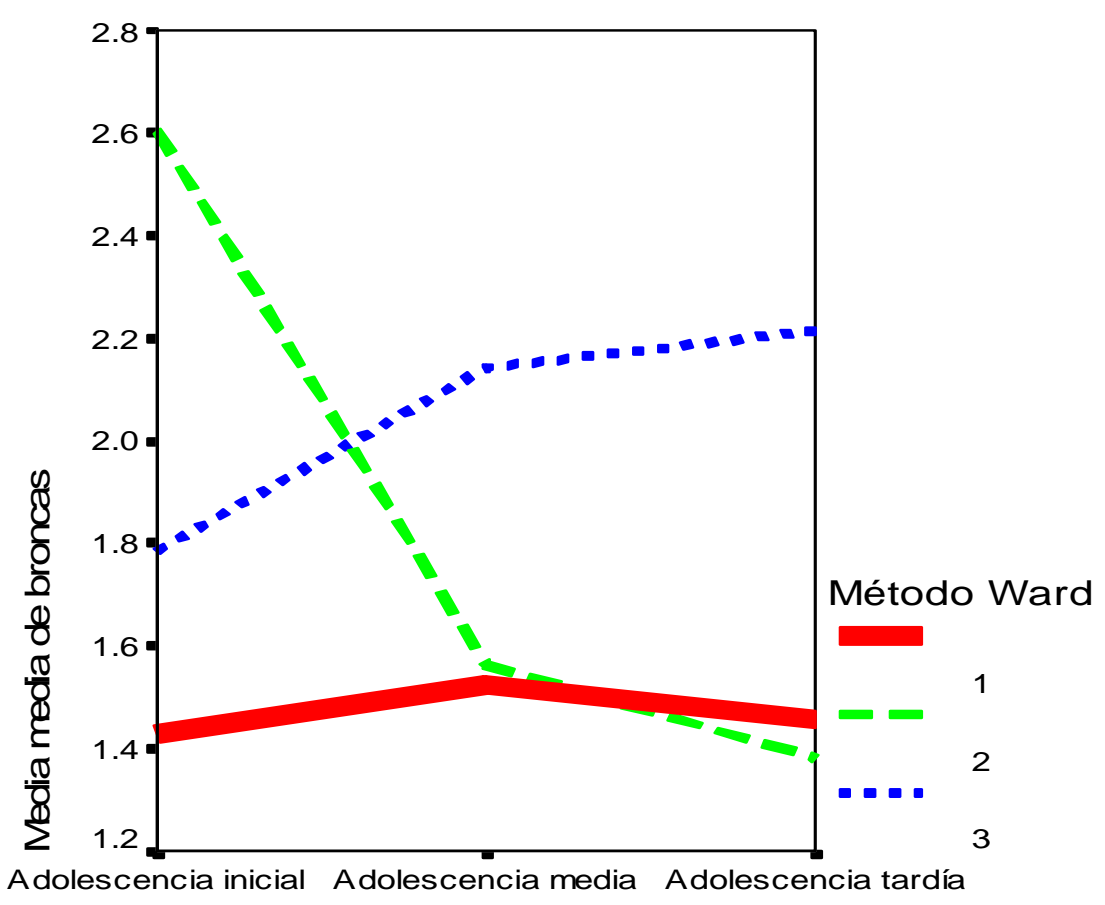

\begin{tabular}{c|c} 
& Frecuencia \\
\hline Grupo 1 & 88 \\
Grupo 2 & 7 \\
Grupo 3 & 6
\end{tabular}


Una mirada longitudinal y transgeneracional sobre los conflictos entre padres y adolescentes 33

Tabla I. Correlaciones entre T1 / T2 / T3 en la frecuencia de los conflictos según la opinión de chicas y chicos.

\begin{tabular}{ccc}
\hline Frecuencia conflictos & Adolescencia inicial - media & Adolescencia media - tardía \\
& $\mathrm{r}$ & $\mathrm{r}$ \\
\hline Chicos & .29 & $\mathbf{. 5 5 * *}$ \\
Chicas & $\mathbf{. 2 9 *}$ & $\mathbf{. 5 2 * *}$ \\
\cline { 2 - 2 } $\mathrm{p}<.05 * \mathrm{p}<.01$ & &
\end{tabular}


$\underline{\text { Una mirada longitudinal y transgeneracional sobre los conflictos entre padres y adolescentes }} 34$

Tabla II. Diferencias entre la adolescencia media y tardía en la frecuencia de los conflictos según la opinión de las madres

\begin{tabular}{lcccc}
\hline & T2 & T3 & $\begin{array}{c}\text { Estab. absoluta } \\
\mathrm{t}\end{array}$ & $\begin{array}{c}\text { Estab. relativa } \\
\end{array}$ \\
& & & $\mathrm{r}$ \\
\hline Conflictos & 1.44 & 1.37 & -1.26 & $\mathbf{. 4 5 * *}$ \\
\hline $\mathrm{p}<.05 * * \mathrm{p}<.01$ & & &
\end{tabular}


Tabla III. Discrepancias en la frecuencia de los conflictos familiares percibidos por madres y adolescentes en la adolescencia media y tardía

\begin{tabular}{lcccccc}
\hline & \multicolumn{3}{c}{ Adolescencia media $(\mathrm{T} 2)$} & \multicolumn{3}{c}{ Adolescencia tardía (T3) } \\
\hline & Madre & Adolescente & $\mathrm{t}$ & Madre & Adolescente & $\mathrm{t}$ \\
Conflictos & 1.44 & 1.53 & $\mathbf{- 2 . 1 5 *}$ & 1.37 & 1.49 & $\mathbf{- 2 . 0 2 *}$ \\
\hline $\mathrm{p}<.05 * * \mathrm{p}<.01$ & & & & & &
\end{tabular}


Este trabajo ha sido realizado gracias a una subvención concedida por el Ministerio de Ciencia y Tecnología dentro de la convocatoria de ayudas para la financiación de proyectos I+D en el marco del Plan Nacional de Investigación Científica, Desarrollo e innovación Tecnológica 2000-2003. Proyecto de investigación con referencia BSO2002-03022. 